\title{
Modelling the Deformation of Biologically Inspired Flexible Structures for Needle Steering
}

\author{
T. Watts ${ }^{1}$ \\ Imperial College London \\ London, UK
}

\author{
R. Secoli ${ }^{2}$ \\ Imperial College London \\ London, UK
}

\author{
F. Rodriguez y Baena ${ }^{3}$ \\ Imperial College London \\ London, UK
}

\begin{abstract}
:
Recent technical advances in minimally invasive surgery have been enabled by the development of new medical instruments and technologies. To date, the vast majority of mechanisms used within a clinical context are rigid, contrasting with the compliant nature of biological tissues. The field of robotics has seen an increased interest in flexible and compliant systems, and in this paper we investigate the behaviour of deformable multi-segment structures, which take their inspiration from the ovipositor design of parasitic wood wasps. These configurable structures have been shown to steer through highly compliant substrates, potentially enabling percutaneous access to the most delicate of tissues, such as the brain. The model presented here sheds light on how the deformation of the unique structure is related to its shape, and allows comparison between different potential designs. A finite element study is used to evaluate the proposed model, which is shown to provide a good fit (root-mean-square deviation $0.2636 \mathrm{~mm}$ for 4-segment case). The results show that both 3-segment and 4-segment designs are able to achieve deformation in all directions, however the magnitude of deformation is more consistent in the 4segment case.
\end{abstract}

Keywords: Mechanical Modelling, Deformable Structures, Biomimetics, Medical Devices, Needle Steering

\section{Introduction}

\section{A. Clinical Motivation}

With the advent of advanced medical devices, traditional open surgical procedures are increasingly being replaced with minimally invasive (MI) alternatives. This shift in surgical approach has been brought about by technical advances in minimally invasive techniques, achieving reduced patient trauma, shorter recovery time, lower chance of infection and reduced scarring [1]. A common MI method is percutaneous intervention, used for clinical access in procedures such as drug delivery, biopsy and brachytherapy, to name but a few. Steerable needles are a promising technology, developed to provide greater targeting accuracy and access to clinical targets which are unreachable via rigid instruments. Crucial to steering these needles, is the ability to predict their behaviour and interaction with soft-tissue.

1t.watts14@imperial.ac.uk

${ }^{2}$ r.secoli@imperial.ac.uk

f.rodriguez@imperial.ac.uk

\section{B. Biological inspiration}

Engineers have often looked to emulate or draw inspiration from biological systems, which via the process of evolution have developed ingenious solutions to the problems of sensing, locomotion, manipulation, etc. In the quest to find a safe place for their offspring, certain parasitic wasps have evolved to possess a long slender channel, called an ovipositor, for depositing their larvae deep within a host. The Sirex noctilio wasp, pictured in Fig. 1 , uses its multi-segment ovipositor to penetrate the bark and bore through the wood of conifers to lay its eggs [2].

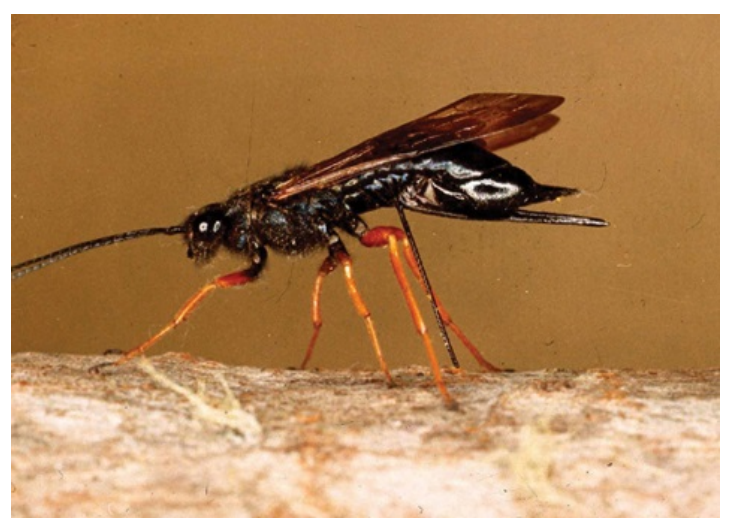

Fig. 1. Woodwasp, Sirex noctilio, boring into wood with its ovipositor to lay eggs. Credit: Natural Sciences Image Library of New Zealand.

Parittotokkaporn et al. [3] were the first to take inspiration from the reciprocating motion of the ovipositor, whereby different segments are inserted alternatively, and developed a needle which was able to achieve tissue traversal with a lower insertion force. Frasson et al. [4] subsequently demonstrated that a 2-segment needle design could perform $2 \mathrm{D}$ steering through a soft substrate with a controllable curvature, and later Secoli et al. demonstrated that 3D steering was achievable with a 4-segment needle. A nonlinear adaptive control strategy was implemented, based on a linear model of the needle, and shown to guide the needle along 3D trajectories [5]. With the increase in number of segments, and complexity of the design, the steering behaviour was no longer well described by the linear model, which motivates the development of a mechanics-based model for the deformation of these unique structures.

\section{Needle steering}

Numerous needle designs have been proposed for steering through soft tissue, as outlined by van de Berg et al. [6]. The steering mechanisms are classified into seven different categories: base manipulation [7], duty-cycled bevel tip (with and without pre-curve) $[8,9,10,11]$, pre- 
curved stylet [12], active cannula [13, 14], programmable bevel tip $[4,15,16]$, tendon actuated tip $[17,18]$ and most recently, optically controlled needle [19].

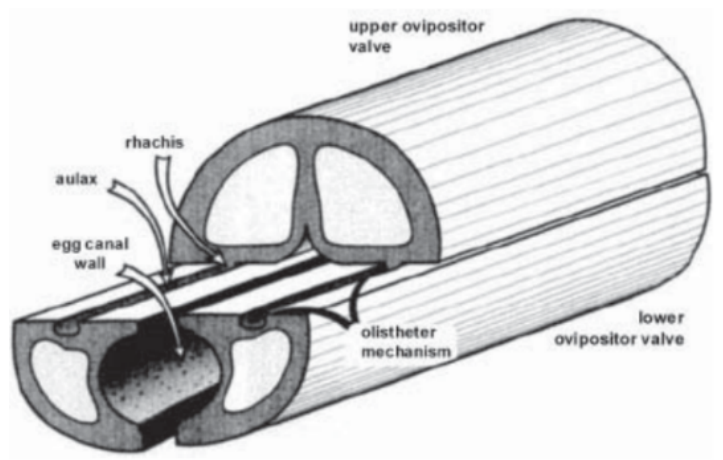

Fig. 2. Structure of the ovipositor, showing the olistheter mechanism which constrains relative motion to the axial direction. [20]

Finite element analysis [21, 22], beam theory [23, 24] and spring foundations [25] have all been used to derive deflection models for steerable needles. Specifically, Oldfield et al. [20] considered the deflection of the programmable bevel-tip needle design, but the analysis is restricted to $2 \mathrm{D}$. The deformation model presented here is the first to consider the complex geometry of the needle design and the effects on the $3 \mathrm{D}$ deformation.

\section{Modelling flexible multi-segment structures}

In previous works, the shape of the needle tip has been shown to control the steering of the needle. The tip shape of an n-segment needle is determined by the relative positioning of the $n$ segments, as shown in Fig. 3. For convenience, each segment's extension is measured relative to a reference plane that moves with the needle tip. These distances are termed the segment offsets and together form an offset vector that describes the tip configuration $o_{i} \in \mathbb{R}^{n}$. It should be noted that this representation has a single redundancy: the common-mode displacement of all segments. In the model presented here, this redundancy is given meaning, with the reference plane notionally describing the boundary between the needle tip, which controls steering, and the needle shaft, which has been shown to approximately follow the trajectory described by the tip.

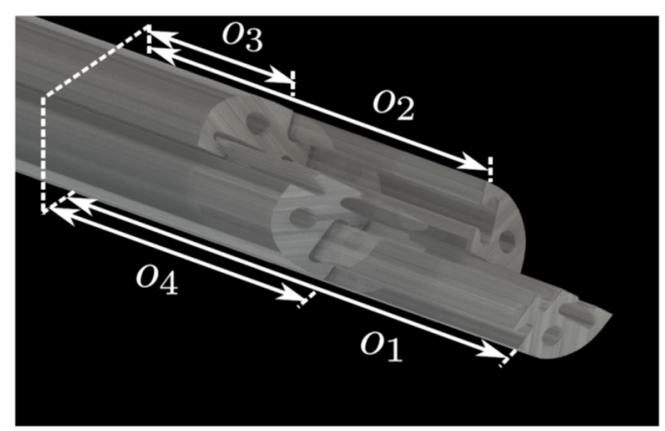

Fig. 3. Rendering of the 4-segment needle showing how the offsets $o_{i}$ control the tip shape.

\section{A. System description}

A 2D representation of the beam-bending problem is shown in Fig. 4 for the 4-segment case. The tip force for each segment $i, \boldsymbol{f}_{i} \in \mathbb{R}^{2}$, is shown acting on the needle tip in the undeformed state. The needle tip is partitioned into portions of constant cross-section. Each portion $j$ has length $l_{j}$ with corresponding second moment of area $\tilde{\mathbf{I}}_{j} \in$ $\mathbb{R}^{2 \times 2}$. The deflection $\boldsymbol{w}_{j} \in \mathbb{R}^{2}$ and rotation $\boldsymbol{\theta}_{j} \in \mathbb{R}^{2}$ of each portion are shown for the needle tip in the deformed state. It is also convenient to define a set $J_{j}$ of segments comprising the portion (e.g. $J_{3}=\{1,2\}$ ). In the following sections, $\mathbf{R}_{\theta} \in S O(2)$ denotes a matrix that represents an anticlockwise rotation of the coordinate system by an angle $\theta$ i.e.

$$
\mathbf{R}_{\theta}=\left[\begin{array}{cc}
\cos (\theta) & \sin (\theta) \\
-\sin (\theta) & \cos (\theta)
\end{array}\right]
$$

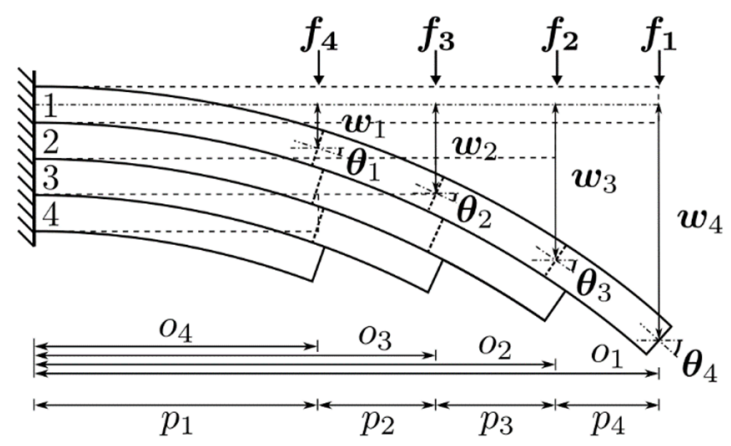

Fig. 4 - 2D representation of the proposed multi-segment deformation model.

\section{B. Tip Forces}

The point load $\boldsymbol{f}_{i}$ acting on each segment is assumed equivalent to the uniform pressure that would be experienced as the needle traverses soft tissue. The axial component is neglected as only transverse deflections are of interest. Figure 5 shows the cross-section of the needle, comprising the four segments, with the respective tip forces shown.

For an $n$-segment needle the expression for the tip force $\boldsymbol{f}_{i}$ in the needle tip coordinate system $\eta$ is as follows:

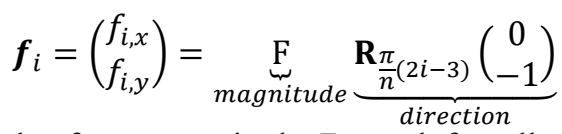

with the force magnitude $F$ equal for all segments. When viewed axially, the forces acting upon the needle are symmetric and sum to zero. The ability to control the direction of deformation is due to structure's configurable nature.
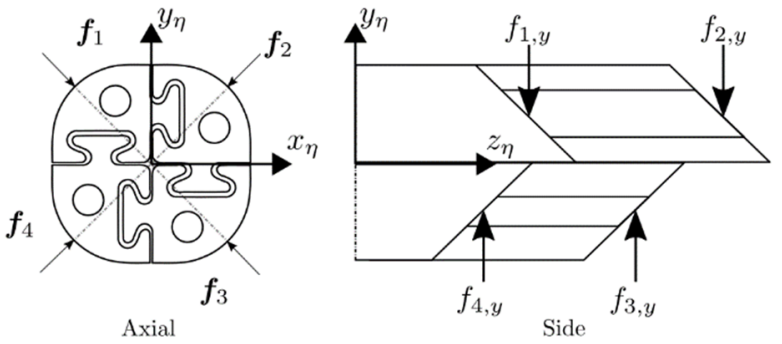

Fig. 5. Axial and side views of the transverse forces acting upon the faces at the tips of the segments. 


\section{Internal reactions}

The second step is to determine the shear force and bending moment acting along the needle tip. Initially, they are calculated at the changes in cross section, given by the portion boundaries. In Figure 7, a 2D representation of a portion $j$ is shown. A virtual cut is considered at the portion boundary and the resultant shear force $s_{j}$ and bending moment $m_{j}$ are shown. As above, for the 3D bending $s_{j}, m_{j} \in \mathbb{R}$ are replaced by the corresponding $\boldsymbol{s}_{j}, \boldsymbol{m}_{j} \in \mathbb{R}^{2}$.

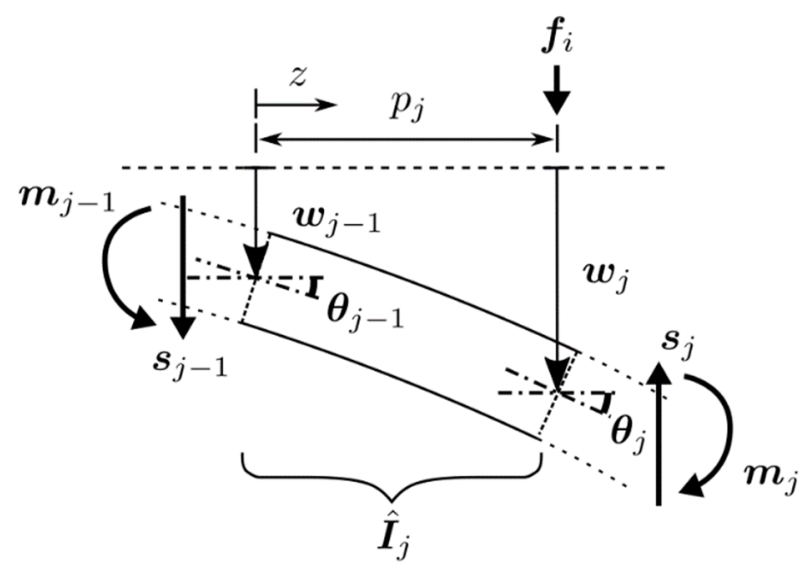

Fig. 6 - Internal bending moment $\boldsymbol{m}_{j}$ and shear force $\boldsymbol{s}_{\boldsymbol{j}}$ shown for portion $\boldsymbol{j}$.

The resultant $\boldsymbol{s}_{j}$ and $\boldsymbol{m}_{\boldsymbol{j}}$ are calculated as follows

$$
\begin{gathered}
\boldsymbol{s}_{j}=\left(\begin{array}{c}
s_{j, x} \\
s_{j, y}
\end{array}\right)=\sum_{J_{j}} \boldsymbol{f}_{i} \\
\boldsymbol{m}_{j}=\left(\begin{array}{l}
m_{j, x} \\
m_{j, y}
\end{array}\right)=\sum_{J_{j}} \mathbf{R}_{\frac{\pi}{2}} \boldsymbol{f}_{i}\left(o_{i}-\sum_{k=1}^{j} l_{k}\right)
\end{gathered}
$$

Note the rotation $\mathbf{R}_{\frac{\pi}{2}}$ is necessary so that a force along the $\mathrm{x}$-axis corresponds to a moment about the $\mathrm{y}$-axis and vice-versa.

For a general cross section, the geometrical contribution to the bending stiffness is given by the second moment of area tensor

$$
\mathbf{I}=\left[\begin{array}{ll}
I_{x x} & I_{x y} \\
I_{y x} & I_{y y}
\end{array}\right]
$$

where $I_{x x}$ and $I_{y y}$ are the second moments of area about the centroidal perpendicular axes $x-x$ and $y-y$ respectively, and $I_{x y}=I_{y x}$ is the corresponding product moment of area.

Assuming that all segments have the same cross section geometry, it is sufficient to calculate the second moment of area tensor for a single segment $\mathbf{I}_{1}$. The transformed second moment of area tensor for any segment $i$ can therefore be calculated as follows

$$
\mathbf{I}_{i}=\mathbf{R}_{\frac{2 \pi}{n}(i-1)} \mathbf{I}_{1} \mathbf{R}_{\frac{2 \pi}{n}(i-1)}{ }^{\mathrm{T}}
$$

Assuming frictionless contact between the segments, the second moment area tensor for portion $j$ is simply equal to the sum of the second moment of area tensors for the comprising segments, i.e.

$$
\widehat{\boldsymbol{I}}_{j}=\sum_{J_{j}} \mathbf{I}_{i}
$$

The Euler-Bernoulli beam equation is as follows

$$
\boldsymbol{m}=\left(\begin{array}{c}
m_{x} \\
m_{y}
\end{array}\right)=-E \mathbf{I} \boldsymbol{\kappa}=-E \mathbf{I}\left(\begin{array}{c}
\kappa_{x} \\
\kappa_{y}
\end{array}\right)=E \mathbf{I} \mathbf{R}_{\frac{\pi}{2}} \frac{\mathrm{d}^{2} \boldsymbol{w}}{\mathrm{d} z^{2}}
$$

where $s$ is length along the beam, $\boldsymbol{\kappa}(z)$ is curvature, $\boldsymbol{m}(z)$ is the bending moment, $\boldsymbol{w}(z)$ is deflection, $E$ is the Young's modulus and $\mathbf{I}$ is the second moment of area tensor.

Rearranging yields

$$
\frac{\mathrm{d}^{2} \boldsymbol{w}}{\mathrm{d} z^{2}}=\frac{1}{E} \mathbf{R}_{\frac{\pi}{2}}{ }^{\boldsymbol{T}} \mathbf{I}^{-\mathbf{1}} \boldsymbol{m}
$$

For $0 \leq z \leq l_{j}$ measured along a single portion $j$, the bending moment as a function of $z$ is

$$
\boldsymbol{m}(z)=\boldsymbol{m}_{j}+\left(l_{j}-z\right) \mathbf{R}_{\frac{\pi}{2}} \boldsymbol{s}_{j}
$$

Substituting (1) into (2), integrating twice with respect to $Z$ and applying continuity conditions at the portion boundaries yields the expressions for the rotation and deflection of each portion.

$$
\begin{aligned}
& \text { For } j=1, \ldots, n \\
& \boldsymbol{\theta}_{j}=\left(\begin{array}{c}
\theta_{j, x} \\
\theta_{j, y}
\end{array}\right)=\left.\frac{d \boldsymbol{w}}{\mathrm{dz}}\right|_{\mathrm{z}=\mathrm{l}_{\mathrm{j}}} \\
& =\underbrace{\boldsymbol{\theta}_{j-1}}_{\text {previous }}+\underbrace{\frac{l_{j}^{2}}{2 E} \mathbf{R}_{\frac{\pi}{2}}{ }^{T} \hat{\mathbf{I}}_{j}^{-1} \mathbf{R}_{\frac{\pi}{2}} \boldsymbol{S}_{j}}_{\text {force }}+\underbrace{\frac{l_{j}}{E} \mathbf{R}_{\frac{\pi}{2}}{ }^{T} \hat{\mathbf{I}}_{j}^{-1} \boldsymbol{m}_{j}}_{\text {moment }} \\
& \boldsymbol{w}_{j}=\left(\begin{array}{c}
w_{j, x} \\
w_{j, y}
\end{array}\right)=\left.\boldsymbol{w}\right|_{\mathrm{z}=\mathrm{l}_{\mathrm{j}}} \\
& =\underbrace{\boldsymbol{w}_{j-1}}_{\text {previous }}+\underbrace{l_{j} \boldsymbol{\theta}_{j-1}}_{\text {rotation }}+\underbrace{\frac{l_{j}^{3}}{3 E} \mathbf{R}_{\frac{\pi}{2}}^{\boldsymbol{T}} \hat{\mathbf{I}}_{j}^{-\mathbf{1}} \mathbf{R}_{\frac{\pi}{2} \boldsymbol{s}_{j}}}_{\text {force }}+\underbrace{\frac{l_{j}{ }^{2}}{2 E} \mathbf{R}_{\frac{\pi}{2}}^{\boldsymbol{T}} \hat{\mathbf{I}}_{j}^{-\mathbf{1}} \boldsymbol{m}_{j}}_{\text {moment }}
\end{aligned}
$$

With fixed boundary conditions at the base:

$$
\boldsymbol{\theta}_{0}=\boldsymbol{w}_{0}=\left(\begin{array}{l}
0 \\
0
\end{array}\right)
$$

The deflection of the furthermost point of the tip is therefore given by $\boldsymbol{w}_{n}$, the model predicted tip deflection.

\section{Finite Element Study}

As a first step to evaluate the validity of the model, Finite Element Analysis (FEA) is performed on the 3segment and 4-segment needle geometries and the observed deflections are compared with model-predicted deflections.

\section{A. System description}

The segments of the structure were meshed with linear hexahedral elements of type C3D8R. A mesh convergence study confirmed stability for deformation with respect to mesh size. For the analysis, the interaction between segments is frictionless and the material is linear elastic with Young's modulus $E$ and Poisson's ration $v$. The second moment of area tensor for a segment's cross-section is $\boldsymbol{I}$. The loading at the tip of each segment is provided by a uniform pressure $p$, acting on the bevelled faces of area $A$, which are cut at an angle $\alpha$. Therefore the equivalent transverse force magnitude, as defined in the model, is $F=$ $p A \cos (\alpha)$. The analysis was performed with Abaqus explicict, using the 'smooth' loading profile and the parameters given in Table 1. Deflection was measured at the tip node of the furthermost extended segment. 
Table 1 - Parameters used in the Finite Element study, along with respective units

\begin{tabular}{|c|c|c|}
\hline Parameter & Value (units) & \\
\hline$E$ & $15 \mathrm{MPa}$ & \\
\hline$\nu$ & 0.33 & \\
\hline$p$ & $0.005 \mathrm{~N} \mathrm{~mm}^{-2}$ & \\
\hline$\alpha$ & $30^{\circ}$ & \\
\hline \multicolumn{3}{|c|}{ for $n=3$} \\
\hline$I_{1}$ & $\begin{array}{cc}-0.2 \overline{5} 8 \overline{2} & -\overline{0} . \overline{11} \overline{5} 5 \\
-0.1155 & 0.3120\end{array}$ & $\mathrm{~mm}^{4}$ \\
\hline $\begin{array}{l}A \\
F_{i}\end{array}$ & $3.015 \mathrm{~mm}^{2}$ & $0.0131 \mathrm{~N}$ \\
\hline \multicolumn{3}{|c|}{$-\ldots$ for $n=4$} \\
\hline$I_{1}$ & $\begin{array}{cc}-0.1 \overline{0} 8 \overline{2} & -\overline{0} . \overline{0} 2 \overline{2} 9 \\
-0.0229 & 0.2147\end{array}$ & $\mathrm{~mm}^{4}$ \\
\hline $\begin{array}{c}A \\
F_{i}\end{array}$ & \multicolumn{2}{|l|}{$0.0095 \mathrm{~N}$} \\
\hline
\end{tabular}

\section{B. Results and discussion}

The resultant tip deformations for the offset configurations trialled are shown below in Table 2 for both the 3-segment and 4-segment needles.

Table 2 - Finite element simulation results, showing axial view of needle tip deflections. An equal load is applied to each segment tip.

\begin{tabular}{|c|c|c|c|}
\hline $\begin{array}{c}\text { Tip } \\
\text { configuration } \\
\text { (mm) } \\
\left\{o_{1}, o_{2}, o_{3}, o_{4}\right\} \\
\end{array}$ & Deflection & $\begin{array}{c}\text { Tip } \\
\text { configuration } \\
(\mathrm{mm}) \\
\left\{o_{1}, o_{2}, o_{3}\right\} \\
\end{array}$ & Deflection \\
\hline $\begin{array}{c}\text { A4: } \\
\{20,20,10,10\}\end{array}$ & & $\begin{array}{c}\text { A3: } \\
\{20,20,10\}\end{array}$ & \\
\hline $\begin{array}{c}\text { B4: } \\
\{20,18,10,10\}\end{array}$ & & $\begin{array}{c}\text { B3: } \\
\{20,18,10\}\end{array}$ & \\
\hline $\begin{array}{c}\mathrm{C} 4: \\
\{20,16,10,10\}\end{array}$ & & $\begin{array}{c}\text { C3: } \\
\{20,16,10\}\end{array}$ & \\
\hline $\begin{array}{c}\text { D4: } \\
\{20,14,10,10\}\end{array}$ & & $\begin{array}{c}\text { D3: } \\
\{20,14,10\}\end{array}$ & \\
\hline $\begin{array}{c}\mathrm{E} 4: \\
\{20,12,10,10\}\end{array}$ & & $\begin{array}{c}\text { E3: } \\
\{20,12,10\}\end{array}$ & \\
\hline $\begin{array}{c}\text { F4: } \\
\{20,10,10,10\}\end{array}$ & & $\begin{array}{c}\text { F3: } \\
\{20,10,10\}\end{array}$ & \\
\hline $\begin{array}{c}\text { G4: } \\
\{20,10,10,12\}\end{array}$ & & $\begin{array}{c}\text { G3: } \\
\{20,10,12\}\end{array}$ & \\
\hline $\begin{array}{c}\mathrm{H} 4: \\
\{20,10,10,14\}\end{array}$ & & $\begin{array}{c}\text { H3: } \\
\{20,10,14\}\end{array}$ & \\
\hline $\begin{array}{c}\text { I4: } \\
\{20,10,10,16\}\end{array}$ & & $\begin{array}{c}\text { I3: } \\
\{20,10,16\}\end{array}$ & \\
\hline $\begin{array}{c}\text { J4: } \\
\{20,10,10,18\}\end{array}$ & & $\begin{array}{c}\text { J3: } \\
\{20,10,18\}\end{array}$ & \\
\hline $\begin{array}{c}\mathrm{K} 4: \\
\{20,10,10,20\}\end{array}$ & & $\begin{array}{c}\text { K3: } \\
\{20,10,20\}\end{array}$ & \\
\hline
\end{tabular}

The tip node deflection for each configuration, $\boldsymbol{w}_{n}$, is shown in Fig. 7 and Fig. 8 for the 3-segment and 4-segment needles respectively, along with the deflection path as the load on each segment is increased from 0 to $F_{i}$.

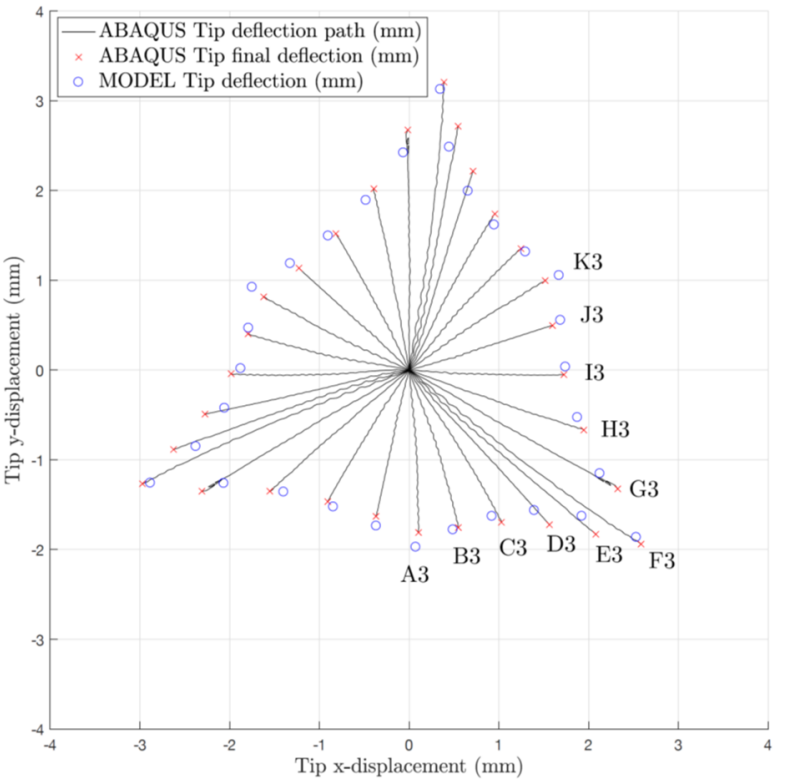

Figure 7 - Comparison between Finite Element simulation results and model predictions for the deflection of 3segment needle tip.

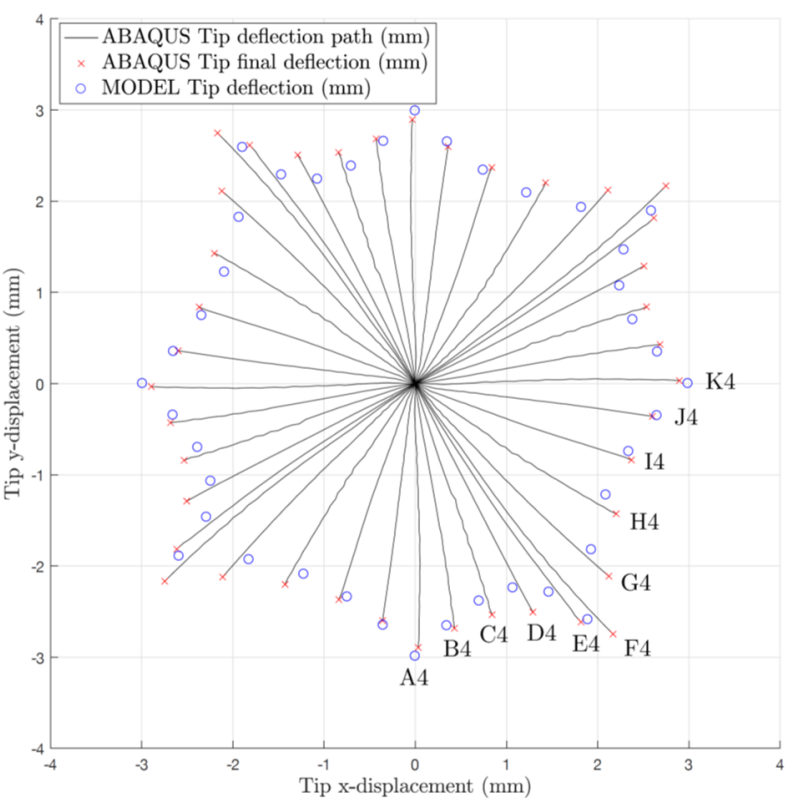

Figure 8 - Comparison between Finite Element simulation results and mode predictions for the deflection of 4segment needle tip.

The root-mean-square deviation (RMSD) between the Finite Element simulation and the model predicted values was $0.1664 \mathrm{~mm}$ and $0.2636 \mathrm{~mm}$ for the 3 -segment and 4 segment cases respectively. Fig. 7 and Fig. 8 both show the increased deflection for offset configurations with either a single or two segments forward, agreeing with previous observation of the needle steering [26]. Additionally, asymmetry due to the needle geometry is observed in both sets of results. The tip deflection paths are "quasi-linear", with non-linearity becoming a greater factor as the deflection increases.

By the comparing the deflection behaviour of the 3segment and 4-segment structures, we can make predictions about how the steering behaviour will differ. 
One of the immediately discernible differences is the variation in deformation magnitude with direction. The ratio of maximum to minimum deflection is 1.703 for the 3 -segment case and 1.235 for the 4-segment case, suggesting that the steering behaviour will be more consistent for the 4-segment design. This is desirable for both control and trajectory planning.

\section{Conclusion}

In this paper, we have presented a model for the deformation of flexible multi-segment structures, which have previously been shown to steer through highly compliant substrates. The model was evaluated via comparison with a set of finite element simulations, performed with 3-segment and 4-segment needle geometries, and was shown to provide a good fit, with a root-mean-square deviation of $0.2636 \mathrm{~mm}$ for the 4segment case.

The results show that the relationship between the configuration of the needle tip and resultant deformation is highly nonlinear, with maximum deformation achieved when a single segment is extended. The results also show how the magnitude of deformation varies with direction, with the 4-segment needle providing more consistent deformation than the 3-segment case, suggesting this design is preferable for applications where multidirectional steering is required.

In future work, we hope to develop a full steering model for the multi-segment needle, based on the deformation model presented here. The model allows us to predict how the needle cross-section affects the system behaviour, and this will be used to inform future design iterations.

\section{Acknowledgment}

This work was supported by the European Union's Horizon 2020 research and innovation Programme under grant agreement No 688279 .

\section{References}

[1] Abolhassani N., Patel R. and Moallem M. Needle insertion into soft tissue: A survey. Medical Engineering and Physics, 29(4):413-431, 2007.

[2] Vincent J.F.V. and King M.J. The mechanism of drilling by wood wasp ovipositors. Biomimetics, 3:187-201, 1995.

[3] Parittotokkaporn T., Frasson L., Schneider A., Huq S. E., Davies B. L., Dagenaar P., Biesenack J. and Rodriguez y Baena F. Soft tissue traversal with zero net force: Feasibility study of a biologically inspired design based on reciprocal motion. In proceedings of the IEEE International Conference on Robotics and Biomimetics Bangkok, Thailand, 2008.

[4] Frasson L., Ko S.Y., Turner A., Parittotokkaporn T., Vincent J.F. and Rodriguez y Baena F. STING: a soft-tissue intervention and neurosurgical guide to access deep brain lesions through curved trajectories, Proc. Inst. Mech. Eng. H., 224(6):775-788, 2010.

[5] Secoli R. and Rodriguez y Baena F. Adaptive path-following control for bio-inspired steerable needles. In proceedings of the 6th IEEE International Conference on Biomedical Robotics and Biomechatronics (BioRob), pp. 87-93, 2016.

[6] van de Berg N.J., van Gerwen D.J., Dankelman J. and van den Dobbelsteen J.J. Design choices in needle steering - a review. IEEE/ASME Trans. on Mechatronics, 20(5):2172-2183, 2015.

[7] Reed K.B., Majewicz A., Kallem V., Alterovitz R., Goldberg K., Cowan N.J. and Okamura A.M. Robot-assisted needle steering. IEEE Robotics Automation Magazine, 18(4):35-46, 2011.

[8] Engh J.A., Minhas D.S., Kondziolka D. and Riviere C.N. Percutaneous intracerebral navigation by duty-cycled spinning of flexible bevel-tipped needles. Neurosurgery, 67(4):1117-1122, 2010.

[9] Kallem V. and Cowan N.J. Image guidance of flexible tip-steerable needles. IEEE Trans. on Robotics, 25(1):191-196, 2009.
[10] Reed K.B., Kallem V., Alterovitz R., Goldberg K., Okamura A.M. and Cowan N.J. Integrated planning and image-guided control for planar needle steering. In proceedings of the IEEE Conference on Biomedical Robotics and Biomechatronics, pp. 819-824, 2008.

[11] Webster R.J., Kim J.S., Cowan N.J., Chirikjian G.S. and Okamura A.M. Nonholonomic modeling of needle steering. The Int. Journal of Robotics Research, 25(5-6):509-525, 2006.

[12] Swaney P.J., Burgner J., Gilbert H.B. and Webster R.J. A flexurebased steerable needle: High curvature with reduced tissue damage. IEEE Trans. on Biomedical Engineering, 60(4):906-909, 2013.

[13]Dupont P.E., Lock J., Itkowitz B. and Butler E. Design and control of concentric-tube robots. IEEE Trans. on Robotics, 26(2):209-225, 2010.

[14]Rucker D.C., Jones B.A. and Webster R.J. A model for concentric tube continuum robots under applied wrenches. In proceedings of the IEEE Int. Conference on Robotics and Automation (ICRA), pp. 10471052, 2010.

[15] Frasson L., Parittotokkaporn T., Davies B.L. and Rodriguez y Baena F. Early developments of a novel smart actuator inspired by nature. In 15th Int. Conference on Mechatronics and Machine Vision in Practice, pp. 163-168, 2008.

[16] Ko S.Y. and Rodriguez y Baena F. Trajectory following for a flexible probe with state/input constraints: An approach based on model predictive control. Robotics and Autonomous Systems, 60(4):509$521,2012$.

[17]Qi P., Liu H., Seneviratne L. and Althoefer K. Towards kinematic modeling of a multi-DOF tendon driven robotic catheter. In proceedings of the 36th Annual International Conference of the IEEE Engineering in Medicine and Biology Society (EMBC), pp. 30093012, 2014.

[18] Roesthuis R.J., van de Berg N.J., van den Dobbelsteen J.J., and Misra $\mathrm{S}$. Modeling and steering of a novel actuated-tip needle through a soft-tissue simulant using fiber bragg grating sensors. In IEEE International Conference on Robotics and Automation (ICRA), pp. 2283-2289, 2015.

[19]Ryu S.C., Quek Z.F., Koh J., Renaud P., Black R.J., Moslehi B., Daniel B.L., Cho K. and Cutkosky M.R. Design of an optically controlled MR-Compatible active needle. IEEE Trans. on Robotics, 31(1):1-11, 2015.

[20]Quicke, D.L.J. and Fitton, M.G. Ovipositor steering mechanisms in parasitic wasps of the families Gasteruptiidae and Aulacidae (hymenoptera). Proc. R. Soc. Lond, 261:99-103, 1995.

[21] Oldfield, M.J., Dini D., Giordano G. and Rodriguez y Baena F. Detailed finite element modelling of deep needle insertions into a soft tissue phantom using a cohesive approach. Computer methods in biomechanics and biomedical engineering, 16(5):530-543, 2013.

[22] Alterovitz R., Goldberg K. and Okamura A.M. Planning for steerable bevel-tip needle insertion through $2 \mathrm{D}$ soft tissue with obstacles. In IEEE International Conference on Robotics and Automation, pp. 1640-1645, 2005.

[23] DiMaio S.P. and Salcudean S.E. Interactive simulation of needle insertion models. IEEE Trans. on Biomedical Engineering, 52(7):1167-1179, 2005.

[24] Khadem M., Rossa C., Usmani N., Sloboda R.S., and Tavakoli M. A Two-Body Rigid/Flexible Model of Needle Steering Dynamics in Soft Tissue. IEEE/ASME Trans. on Mechatronics, 21(5):2352-2364, 2016.

[25] Glozman D. and Shoham M. Flexible needle steering and optimal trajectory planning for percutaneous therapies. In proceedings of the International Conference on Medical Image Computing and Computer Assisted Intervention, pp. 137-144, 2004.

[26] Burrows C., Secoli R. and Rodriguez y Baena F. Experimental characterisation of a biologically inspired 3D steering needle. Proceedings of the International Conference on Control, Automation and Systems, pp. 1252-1257, 2013. 